\title{
lipoescultura no tumescente
}

\section{Non tumescent liposculpture}

\author{
Cahuana Salinas, E.R.*, Solís Gonzales, M.**, Cáceres Canales, L.***, Seminario Valle, F.***k*
}

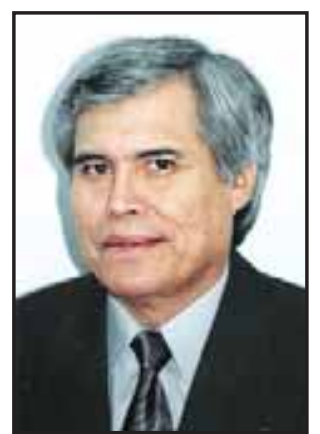

Cahuana Salinas, E.R.

\section{Resumen}

En este trabajo, debido a los serios cuestionamientos que tenemos con el "dogma" de la "Tumescencia" como requisito para hacer la liposucción convencional, planteamos como alternativa una nueva técnica denominada por nosotros "lipoescultura no tumescente" que se basa en dos principios: 1) uso de solución vasoconstrictora $2 \mathrm{~A}, 2$ ) Técnica de infiltración suprafascial. Nuestro trabajo es de tipo prospectivo y es el producto de 18 meses de observación, análisis y puesta en marcha de una hipótesis que finalmente confirmó nuestras sospechas: reducción muy importante de los riesgos de una lipoescultura, resultados estéticos de mejor calidad y convicción de lo innecesario y poco racional que, a nuestro juicio, resulta la tumescencia en la lipoescultura; por lo tanto creemos que su vigencia está seriamente cuestionada en estos momentos. Sin embargo debemos reconocer la importancia que tuvo en tanto no hubo un cuestionamiento serio y una alternativa razonable.

\section{Palabras clave Liposucción. Técnica tumescente. Lipo- escultura.}

Código numérico 104-5311

\author{
Key words Liposuction. Lipoesculpture. Tumescent \\ technique.
}

Código numérico 104-5311
In this work, due to the serious questions that we have with the "dogma" of "Tumescencia" as a requirement to make the conventional liposuction, we suggest as an alternative a new technique named by us "non tumescent liposcultpture", based in two principles: 1) use of solution 2A, 2) suprafascial infiltration. Our work is prospective and it is the result of 18 months observation, analysis and development of a hypothesis that has finally confirmed our suspicions: very important reduction of the risks of a liposculture, aesthetic results of better quality and the conviction about how unnecessary and not very rational "tumescencia" can be in the liposculture; therefore we believe that its validity is seriously questioned now, however we should recognize the importance that it had as long as there were not serious questions nor a reasonable alternative.

\footnotetext{
Cirujano Plástico y Médico Internista. 


\section{Introducción}

La lipoescultura ha alcanzado una gran importancia en nuestra especialidad no solo por los beneficios que logra y el volumen de operaciones que se están realizando, sino también por toda la controversia que se origina entre el público cada vez que este procedimiento toma un curso dramático.

En el momento actual la liposucción convencional se realiza después de haber recurrido al artificio dogmático de la tumescencia (1), lo cual significa que la cantidad de solución de Klein que se usa necesariamente esté en relación directa con el volumen de tejido adiposo que se intenta extraer, por lo tanto es abundante (2).

La tumescencia fue motivo por nuestra parte de los siguientes cuestionamientos:

- La administración de grandes volúmenes de solución de Klein (agua, cloruro de sodio, y adrenalina) supone un riesgo en aquellos pacientes que tienen una reserva funcional cardiopulmonar disminuida.

- El recurso de la tumescencia enmascara el real espesor del colgajo que estamos dejando al practicar una liposucción, lo cuál hace más probable las secuelas en forma de irregularidades la piel, sobre todo en la superficie anterior del abdomen.

- El recurso de la tumescencia provoca el llamado signo del tonel, puesto que enmascara los volúmenes y las formas de las zonas operatorias.

- El tejido adiposo aspirado durante la liposucción está muy hidratado, y los cálculos de volumen del mismo son poco reales cuando lo usamos para inyectarlo como injerto, o simplemente cuando queremos medir el volumen real de grasa extraído.

Todas estas cuestiones arriba mencionadas nos indujeron a plantear una alternativa, la lipoescultura no tumescente que pasamos a describir a continuación.

\section{Material y método}

Nuestra casuística está constituida por 72 pacientes que pertenecen a la práctica privada de los cirujanos plásticos integrantes del grupo TAGLIACOZZI, en los que se practicó lipoescultura del tronco y caderas. Las intervenciones se realizaron durante el período comprendido entre enero del 2001 y junio del 2002. Los detalles relativos a la casuística son:

- Pacientes femeninos 68 (94.44\%); pacientes masculinos $4(5.55 \%)$.

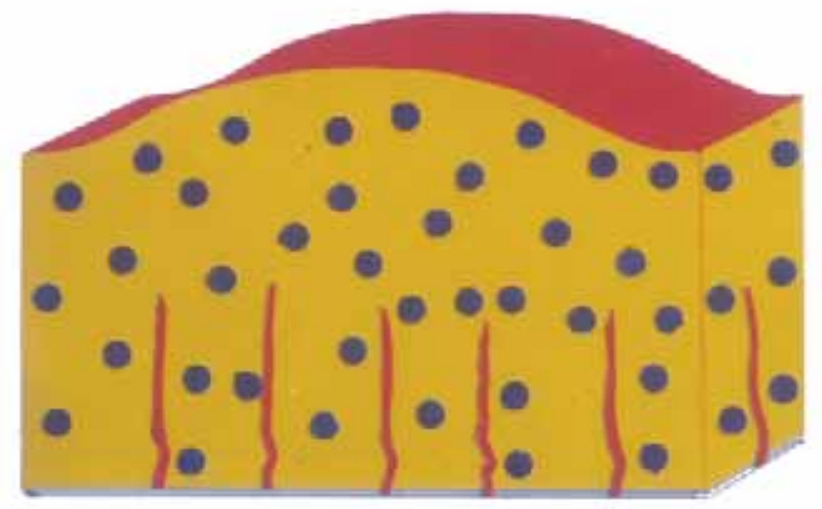

Figura 1. Representación de un colgajo dermograso con infiltración tumescente, mostrando el engrosamiento del colgajo. Los puntos azules representan la solución de Klein.

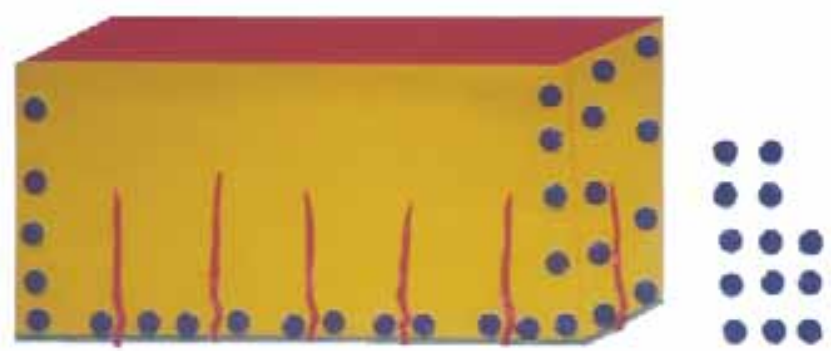

Figura 2. Representación de un colgajo dermograso con la técnica de infiltración suprafascial. Los puntos azules representan la solución 2A.

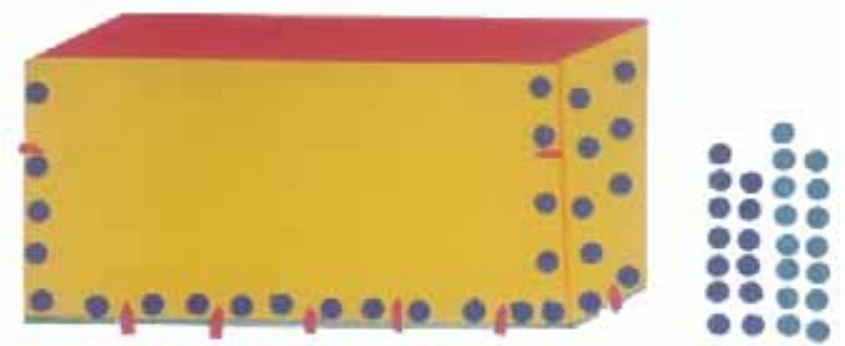

Figura 3. El dibujo representa el eficiente efecto vasoconstrictor de la infiltración suprafascial.

- Las edades estuvieron comprendidas entre los 19 y los 50 años.

- Se practicó anestesia peridural en el $100 \%$ de los casos.

- Las intervenciones se practicaron de forma ambulatoria en 28 pacientes $(38.8 \%$ ) y con un día de hospitalización en 44 pacientes $(61.2 \%)$.

- El exceso de peso que presentaban los pacientes fue del 1 al $70 \%$ sobre el peso ideal.

\section{Técnica operatoria}

Nuestra técnica operatoria tiene dos principios:

1. Solución vasoconstrictora 2 A (Sol. 2 A) 


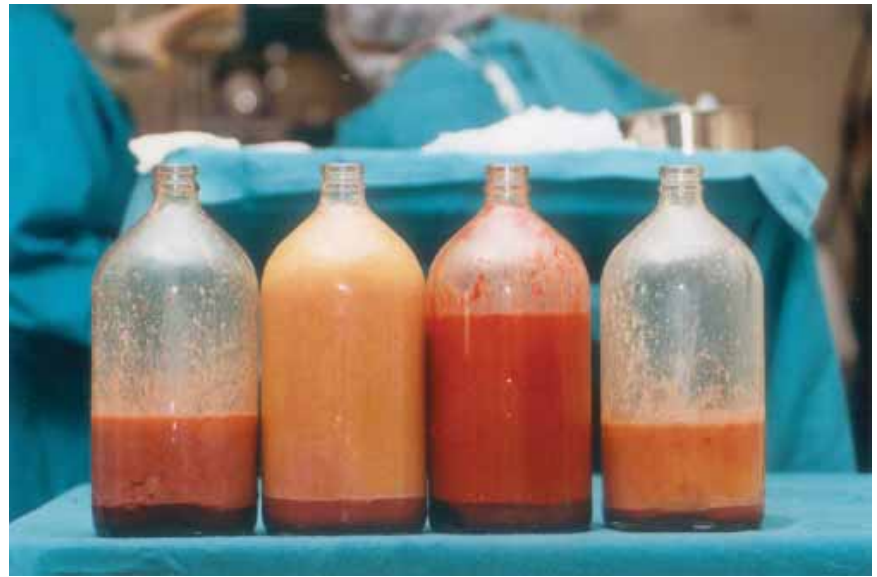

Figura 4: Producto de la lipoaspiración de todo el tronco en una paciente, sin ninguna manipulación; en el fondo de cada frasco podemos ver el líquido sanguinolento decantado.

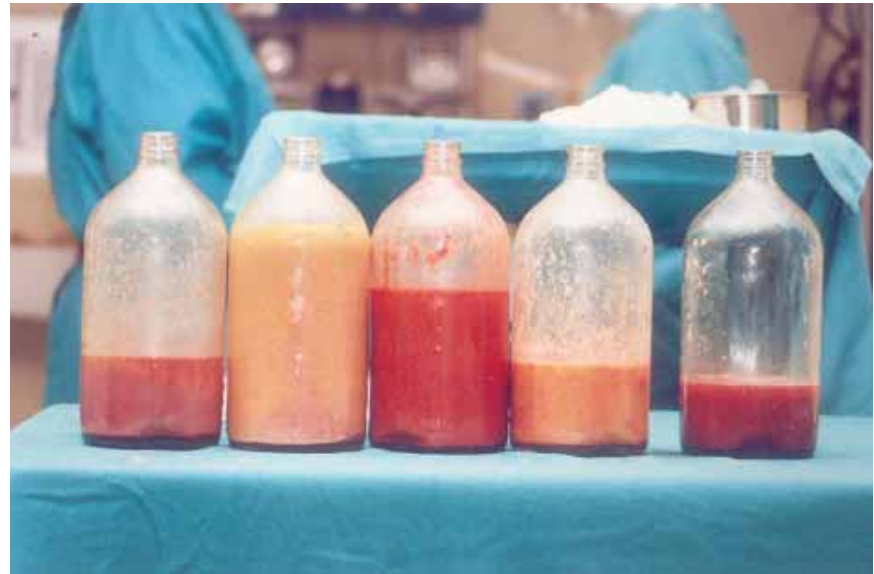

Figura 5: El líquido decantado es aislado en un quinto frasco para poder hacer los mediciones de Hb. (Frasco de la derecha).

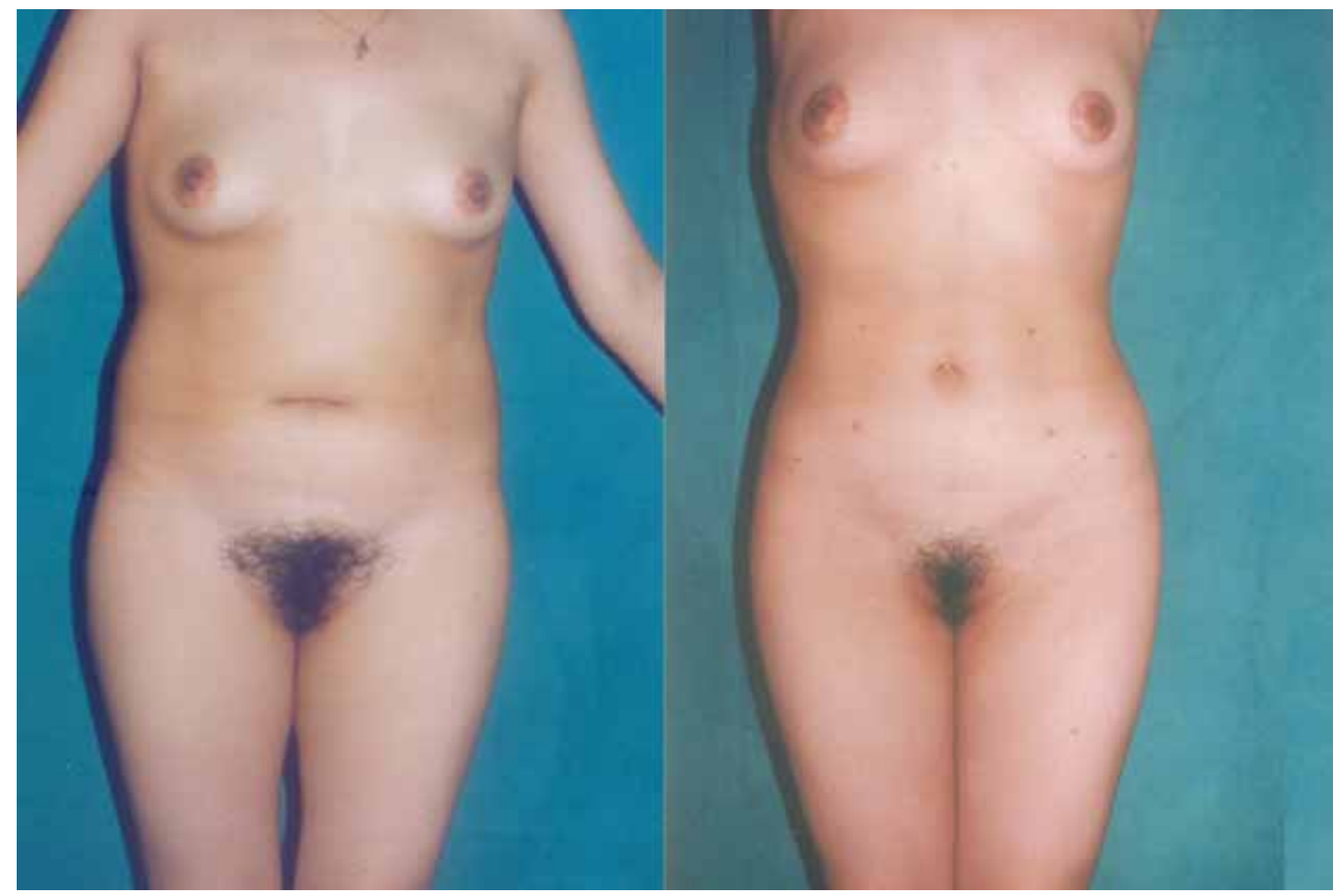

Figura 6: Paciente de 23 anos sometida a lipoescultura no tumescente del tronco y caderas con injertos de tejido adiposo en región glútea y en superficie interna de muslos; resultado a los 4 meses.

a. Suero fisiológico. $1 \mathrm{~L}$.

b. Adrenalina. $2 \mathrm{mg}$.

2. Técnica de infiltración suprafascial.

La Sol. 2 A es usada a temperatura ambiente: En cuanto al segundo principio, hay que precisar que la técnica de infiltración suprafascial consiste en que la Sol 2 A se infiltra en toda la zona operatoria, en el plano estrictamente suprafascial, pero en los límites de la zona operatoria y solamente en ellos, la infiltración se hace en todo el espesor del colgajo, de tal modo que quede bloqueado todo aporte san- guíneo a la zona operatoria (Fig. 1-3). Esta infiltración se realiza en dos fases: en la primera se infiltra la superficie anterior y los laterales del tronco y en la segunda fase se infiltra la superficie posterior $\mathrm{y}$ las caderas.

\section{Resultados}

El producto lipoaspirado fue en promedio $2598 \mathrm{ml}$., con un máximo de 4000 y un mínimo de $1150 \mathrm{ml}$.

La calidad del tejido adiposo corresponde al tipo biopsia (Fig. 4). 

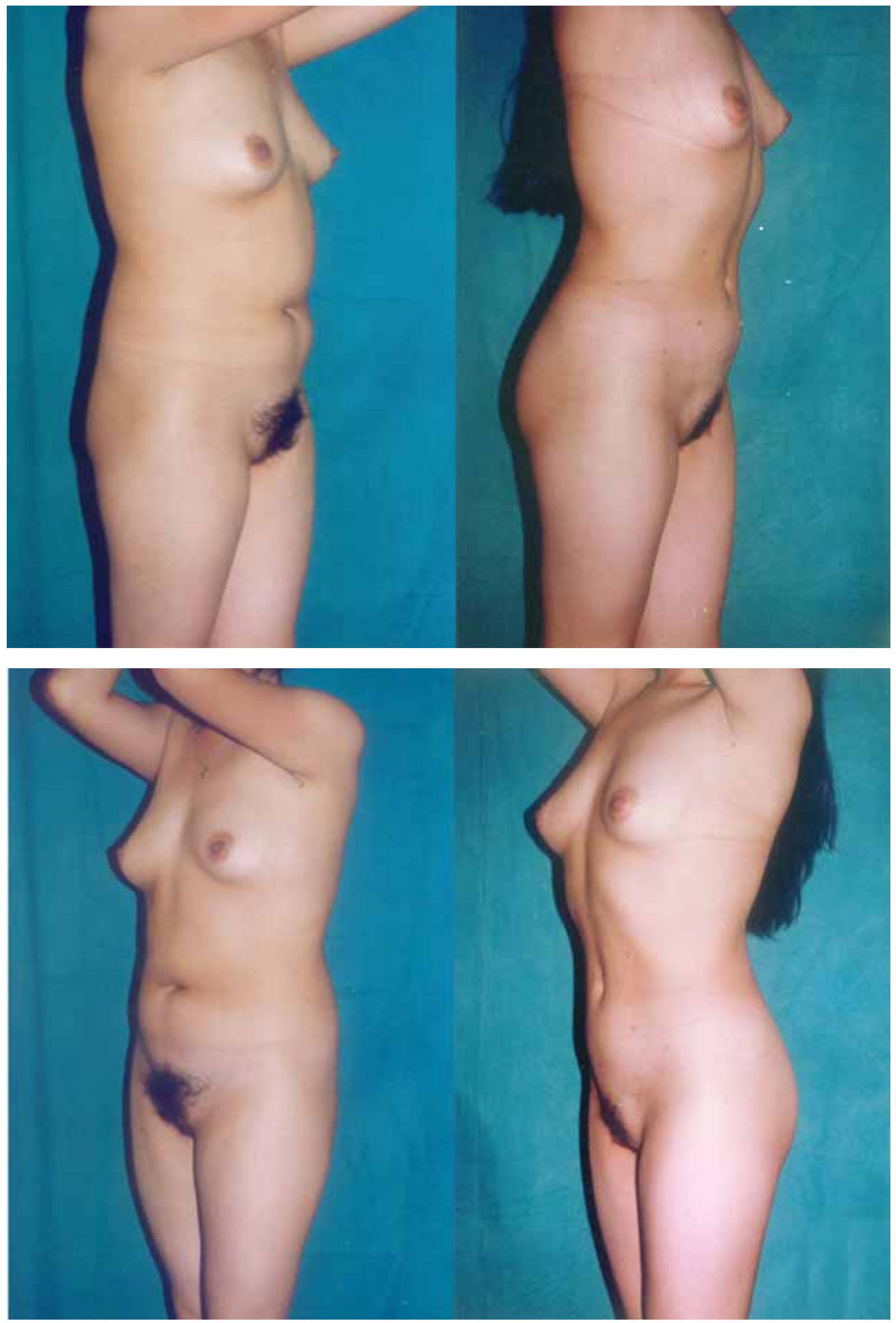

Figuras 7 y 8: La misma paciente para apreciar la regularidad de la superficie anterior del abdomen y el beneficio del injerto adiposo en región glútea. 


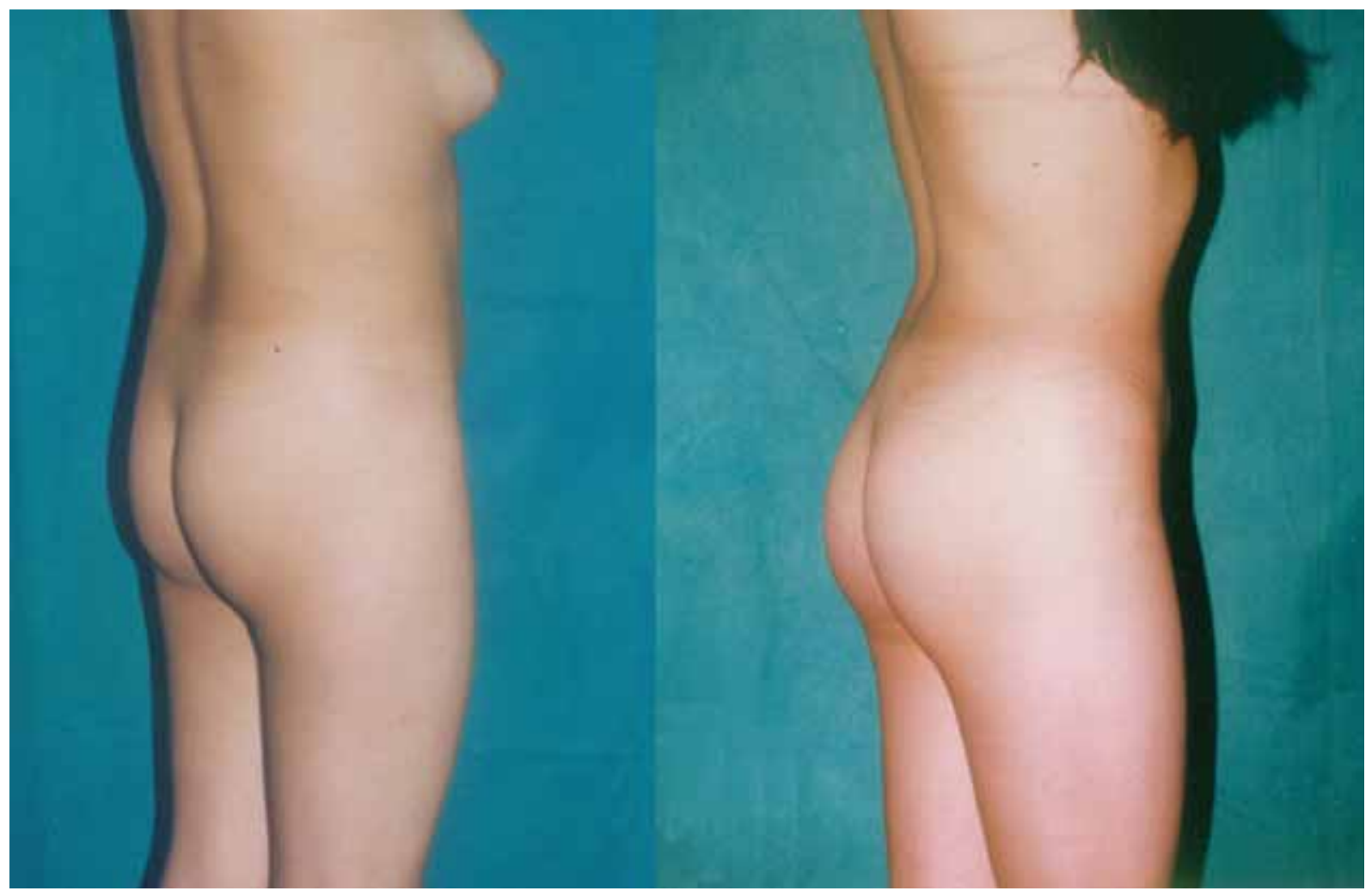

Figura 9: La misma paciente mostrando los beneficios del injerto en región glútea.

El volumen del líquido decantado fue en promedio $510 \mathrm{ml}$. (Fig. 5), y la medición de hemoglobina en el mismo fue en promedio $1.02 \mathrm{grs} \%$ con un mínimo de 0.3 grs $\%$ y un máximo de 2.3 grs $\%$ en un solo caso. La hemoglobina que se pierde en el líquido decantado equivale a un volumen de $43.75 \mathrm{ml}$. de sangre de media, cantidad que se multiplica por un factor de corrección de 2 para estimar incluso la pérdida de sangre que está mezclada con el tejido adiposo y que no se ha decantado; por lo tanto el promedio de "sangre visible" que se pierde es de $87 \mathrm{ml}$., con un mínimo de $20.3 \mathrm{ml}$ y un máximo de $218 \mathrm{ml}$. en un sólo caso.

La cantidad de solución vasoconstrictora 2 A necesaria para una lipoescultura de tronco (incluyendo superficie anterior, posterior, laterales y caderas) fue en promedio $1680 \mathrm{ml}$. con un mínimo de $1420 \mathrm{ml}$. y un máximo de $1900 \mathrm{ml}$.

La cantidad de Adrenalina necesaria para una lipoescultura de tronco y caderas fue en promedio $3.3 \mathrm{mg}$. con un mínimo de $2.8 \mathrm{mg}$. y un máximo de $3.8 \mathrm{mg}$.

La piel de la zona operatoria no presentó irregularidades en el postoperatorio.

Parámetros hemodinámicos: la tensión arterial se mantuvo dentro de límites normales y el pulso, durante y al final de la primera fase de la infiltración con Sol. 2 A se mantuvo en un rango de 70 a 90 pulsaciones por minuto.

El $38.8 \%$ de los pacientes regresaron a su domicilio después de superar el efecto anestésico y lo hicieron en condiciones hemodinámicas adecuadas. El
$61.2 \%$ de los pacientes dejaron la Clínica entre las 18 y 24 horas siguientes al acto operatorio.

Discusión

A nuestro modo de ver las virtudes de la técnica de Lipoescultura no tumescente pueden ser demostradas en pacientes en quienes se intente aspirar grandes, medianos, o pequeños volúmenes de tejido adiposo.

La calidad del tejido adiposo aspirado es de tipo biopsia, y si adicionalmente existe la intención de hacer injertos de este tejido, las condiciones que presenta este tejido de tipo biopsia no pueden ser superadas por las obtenidas mediante ninguna técnica de infiltración.

La cantidad de sangre visible que se pierde con nuestra técnica en una lipoescultura de todo el tronco y caderas es en promedio $87 \mathrm{ml}$., lo cual es una cantidad muy aceptable para un procedimiento de esta envergadura; la disminución de Hb.en sangre es de 2 gr. \% medida a las 24 horas del acto operatorio, y en ningún caso se administró transfusión de sangre o coloides (Gráfico 1), a diferencia de lo referido por Cárdenas (3), que indica disminuciones de 3,8 gr. \% de hemoglobina como promedio y de las referencias de Ferrari y Fournier (2) que señalan entre 2 y 3 gr. $\%$ de déficit de hemoglobina a las 24 horas.

El volumen del líquido decantado fue en promedio $510 \mathrm{ml}$., constituyendo en promedio 1/5 del volumen total de la lipoaspiración, lo cual es un hallazgo muy 


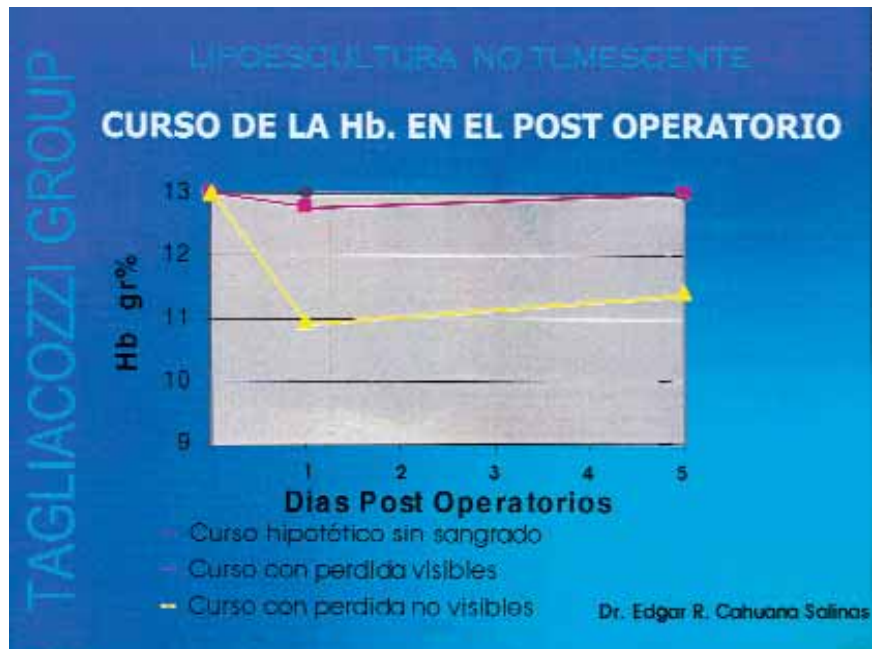

Gráfico 1.

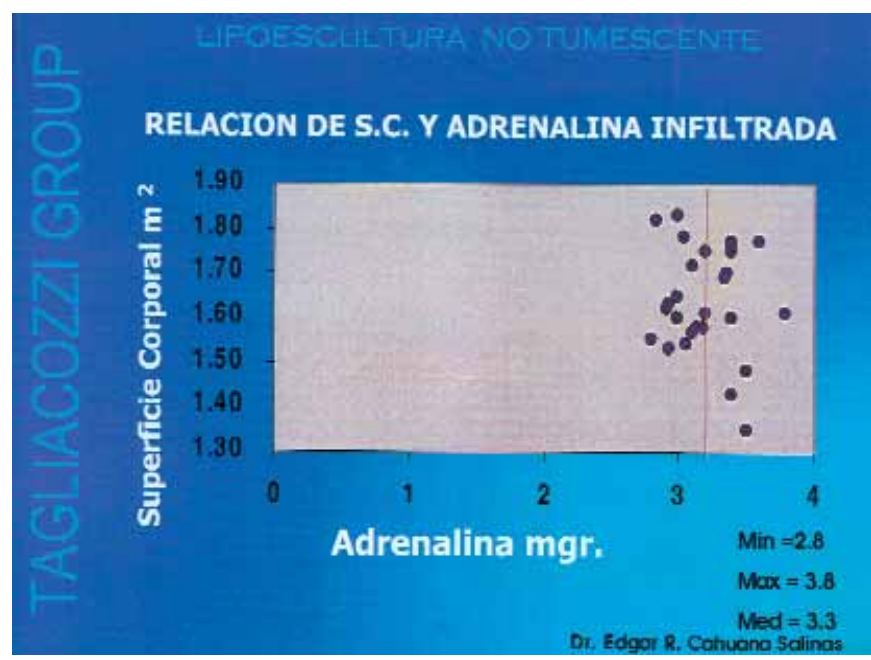

Gráfico 3.

similar cuando se hace uso de la técnica tumescente; por lo tanto los usuarios de la tumescencia están obligados a hacer una reflexión aritmética que sería la siguiente: si el volumen del liquido decantado es "igual" tanto cuando se hace una infiltración no tumescente (muy poco volumen) como cuando se hace una infiltración tumescente (abundante volumen) ¿a qué espacio llegó la diferencia de los volúmenes de infiltración?; pues aproximadamente $70 \%$ de líquido subcutáneo será absorbido eventualmente (4).

Cuando evaluamos el volumen de la Solución 2 A que se necesita para una lipoescultura de tronco en relación a la superficie corporal de la paciente, encontramos que por más que la superficie corporal varíe en un rango muy amplio, el volumen de Sol. 2 A requerido tiene un rango de variación muy estrecho que va desde un mínimo de $1420 \mathrm{ml}$ a un máximo de 1900 $\mathrm{ml}$. y cuyo promedio es de solamente $1680 \mathrm{ml}$. (Gráfico 2), a diferencia de la técnica tumescente que en un abdomen puede inyectar entre 7-8 litros de solución de Klein (2).

Cuando evaluamos la cantidad de mg. de adrenalina

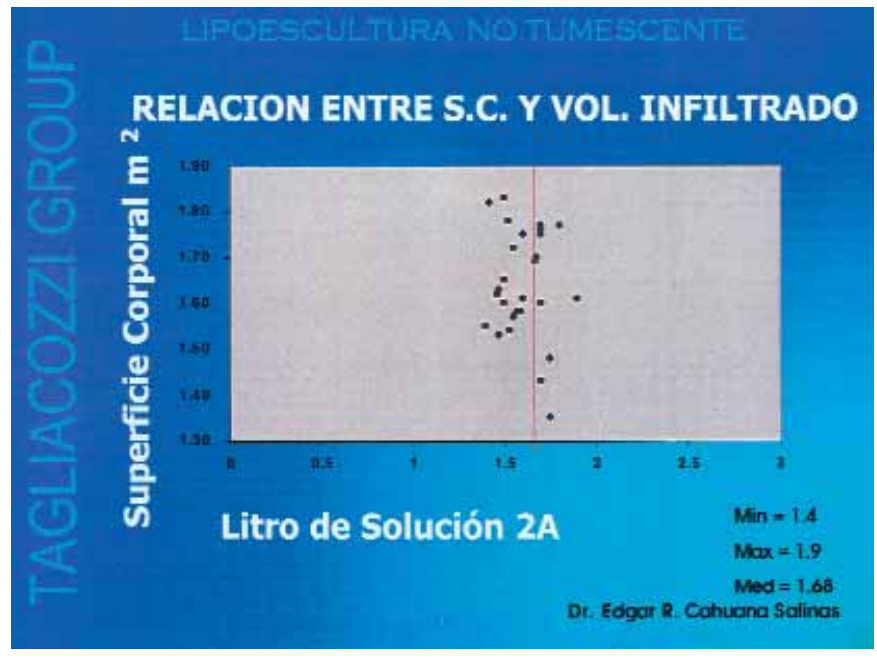

Gráfico 2.

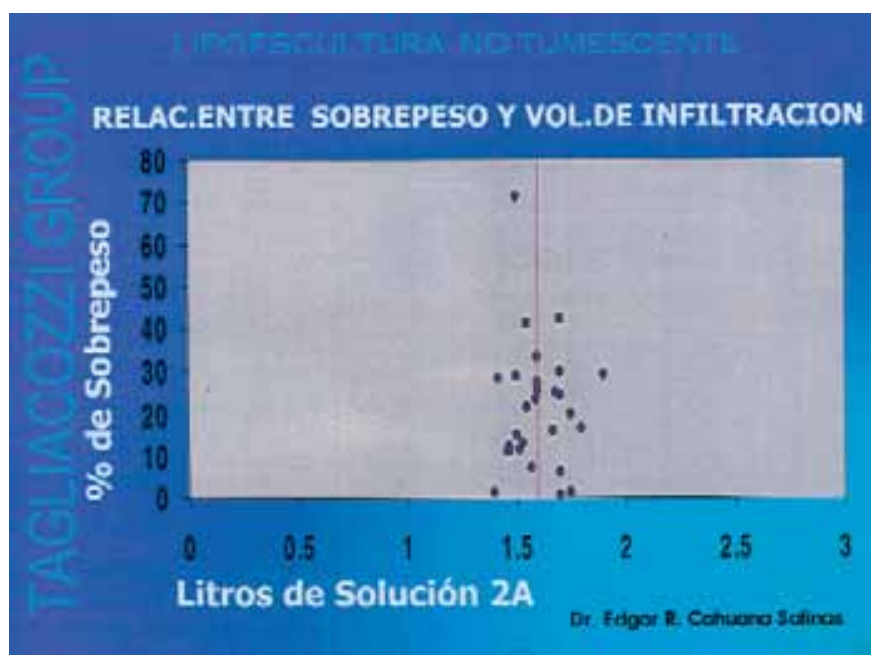

Gráfico 4.

necesarios para la lipoescultura en relación a la superficie corporal (5) se encuentra similar reflexión planteada en el párrafo anterior: por más que la superficie corporal varíe en un rango muy amplio, la cantidad de adrenalina requerida tiene un rango de variación muy estrecho que va desde una mínima de $2.2 \mathrm{mg}$. a una máxima de $3.8 \mathrm{mg}$. y un promedio de $3.3 \mathrm{mg}$. solamente (Gráfico 3), en contraste con la técnica tumescente cuya dosis total puede llegar a $12 \mathrm{mg}$. (4).

Cuando evaluamos la cantidad de solución 2 A requerida para la infiltración en una lipoescultura en relación al \% de sobrepeso de los pacientes encontramos que, por más que el parámetro sobrepeso tenga un rango de variación muy amplio, la cantidad de solución $2 \mathrm{~A}$ requerida tiene un rango de variación muy estrecho que va desde 1420 a $1900 \mathrm{ml}$. con un promedio de $1680 \mathrm{ml}$. (Gráfico 4).

La piel de la zona operatoria, prácticamente no presenta irregularidades por retracción debido a que el espesor del colgajo que queda en el acto operatorio es "real" a diferencia de los casos en los que se recurre a la tumescencia, en los que el espesor del colgajo está 
enmascarado por la infiltración; por lo tanto cuando desaparece el líquido infiltrado durante el post-operatorio inmediato y el edema en el post-operatorio mediato, es mucho más probable que aparezcan las irregularidades por retracción cicatricial, que no desaparecerán por más que nos engañemos con los masajes, el ultrasonido o cremas diversas (Fig 6-9).

Contrariamente a lo que el sentido común del médico puede dictar, durante la primera fase de la infiltración de la Solución 2A (900 ml en promedio), la frecuencia cardiaca se mantiene en valores sorprendentemente bajos, entre 70 y 90 latidos por minuto, lo cual significa que la acción de la adrenalina es inmediata y no hay más que una absorción muy escasa al torrente sanguíneo.

Nuestra técnica de lipoescultura no tumescente ejecutada adecuadamente, puede permitir perfectamente tratar de forma totalmente ambulatoria a todos aquellos pacientes desprovistos de alguna enfermedad preoperatoria concomitante.

\section{Conclusiones}

Nuestro trabajo intenta demostrar que la lipoescultura no tumescente es una técnica muy simple, pero sobre todo la más racional, ya que con la Solución 2A y la técnica de infiltración estrictamente suprafascial tiene la principal virtud de administrar cantidades increíblemente mínimas de Cloruro de Sodio, Adrenalina y Agua, con lo cuál se disminuyen al mínimo las alteraciones hemodinámicas por sobrecarga de fluidos y drogas, a la vez que se reduce significativamente el sangrado intraoperatorio.
Una virtud importante de esta técnica es la poco probable secuela de irregularidades postoperatorias en la piel de la zona operada, porque el espesor del colgajo quedará tal cual lo deje el cirujano, al no estar enmascarado por la infiltración.

El tejido adiposo aspirado, al tener caracteres de biopsia, tiene las mejores condiciones para ser usado como injerto en otras zonas.

Finalmente estamos en condiciones de afirmar que no existe justificación para recurrir al dogma de la tumescencia en la práctica de la lipoescultura convencional, que ante nuestra alternativa, resulta innecesario y poco racional.

\section{Dirección del autor}

\author{
Dr. Edgar R. Cahuana Salinas \\ Julio C. Tello 356 - D Lince \\ Lima (Perú) \\ e-mail: Cahuana_salinas@hotmail.com
}

\section{Bibliografía}

1. Rovira R.V. Serra J. M. "Liposucción en Cirugía Plástica y Estética” Edición: 1987 Salvat.España.

2. Ferrari F. Pitanguy I. "Cirugía Estética" 1 Edición 1999. Actualidades Médico-Odontológicas.Impreso en Colombia pp. 367 - 401.

3. Cardenas L. Gonzales L.C. Tobar A. Lacouture A. "Liposucción circunferencial de grandes volúmenes sola o combinada para lograr mejoría del contorno corporal”. Anales del XIII Congreso de la Federación IberoLatinoamericana de Cirugía Plástica”. 2000. 1. pp 135-146.

4. Rohrich R.J. Beran S.J.Kenkel J.M. "Lipoescultura" $1^{\circ}$ Edición: año 2001.Actualidades Médico-Odontológicas. Impreso en Colombia.

5. Houssay B.A. "Fisiología Humana" 4 Edición : 1969. Ateneo; Argentina pp 497-502. 